Original paper

\title{
Investigation of the magnetic susceptibility properties of fresh and fixed mouse heart, liver, skeletal muscle and brain tissue
}

\author{
Jan Klohs ${ }^{\text {a,b, }}$, Ann M. Hirt ${ }^{c}$ \\ ${ }^{a}$ Institute for Biomedical Engineering, University of Zurich and ETH Zurich, CH-8093 Zurich, Switzerland \\ ${ }^{\mathrm{b}}$ Zurich Neuroscience Center, University of Zurich, Zurich, Switzerland \\ ${ }^{\mathrm{c}}$ Institute of Geophysics, ETH Zurich, CH-8092 Zurich, Switzerland
}

\section{A R T I C L E I N F O}

\section{Keywords:}

Magnetic susceptibility

Magnetic resonance imaging

Mouse organ tissue

Fixation

Paraformaldehyde

\begin{abstract}
A B S T R A C T
Purpose: Several magnetic resonance imaging (MRI) techniques exploit the difference in magnetic susceptibilities between tissues, but systematic measurements of tissue susceptibility are lacking. Furthermore, there is the question as to whether chemical fixation that is used for ex vivo MRI studies, affects the magnetic properties of the tissue. Here, we determined the magnetic susceptibility and water content of fresh and chemically fixed mouse tissue.

Methods: Mass susceptibility of brain, heart, liver and skeletal muscle samples were determined on a vibrating sample magnetometer at room temperature. Measurements at 50, 125, 200 and $295 \mathrm{~K}$ were performed to assess the temperature dependence of susceptibility. Moreover, we measured water content of fresh and fixed samples. Results: All samples show mass susceptibilities between -0.068 and $-1.929 \times 10^{-8} \mathrm{~m}^{3} / \mathrm{kg}$, compared to -9.338 $\times 10^{-9} \mathrm{~m}^{3} / \mathrm{kg}$ of double distilled water. Heart tissue has a more diamagnetic susceptibility than the other tissues. Compared to fresh tissue, fixed tissue has a less diamagnetic susceptibility. Fixed tissue was not different in water content to fresh tissue and showed no consistent dependence of susceptibility with temperature, whereas fresh tissue shows a decrease to at least $125 \mathrm{~K}$, indicative of a paramagnetic component.

Conclusions: Biological tissues are diamagnetic in comparison to water, where the heart is more diamagnetic than the other tissues, with paramagnetic contributions. Fixation rendered tissue less diamagnetic compared to fresh tissue. Our measurements revealed differences in tissue susceptibility between VSM and QSM, inviting more research to compare susceptibility-based MRI methods with physical measurements of tissue susceptibility.
\end{abstract}

\section{Introduction}

Magnetic susceptibility is a fundamental electromagnetic property that measures the ability of a substance to become magnetized when placed in an external magnetic field. Many biological tissues exhibit either positive or negative susceptibility, and are termed paramagnetic or diamagnetic, respectively. The fact that different biological tissues and cellular structures have different magnetic susceptibilities has been exploited for contrast generation in magnetic resonance imaging (MRI). Using $\mathrm{T}_{2}$ *-weighted gradient echo imaging, variations of tissue magnetic susceptibility typically lead to local signal cancellations in magnitude images and non-local changes in phase images due to the induced frequency shifts. The combination of magnitude and the phase information into a single image, the so-called susceptibility-weighted image, has been shown to significantly enhance image contrast qualitatively between tissues of different magnetic susceptibility [1]. With the introduction of quantitative susceptibility mapping (QSM), a postprocessing technique for gradient echo data, quantitative maps of bulk tissue magnetic susceptibility of the human brain and body can be obtained. QSM recovers the susceptibility distribution from the measured local field (i.e. phase) distribution [2-8]. A multitude of clinical and preclinical studies illustrated how QSM can be applied in vivo; for example, to detect vascular abnormalities, visualize blood products and calcifications; microstructural changes and lesions, or map abnormalities in iron metabolism [9-14]. These studies have collectively demonstrated that pathological alterations in the brain, heart, muscle and abdomen in various diseases are associated with changes of tissue magnetic susceptibility. For QSM, algorithms are required for the numerical solution of the field-to source inverse problem and susceptibility maps computed with different algorithm have shown substantial

\footnotetext{
* Corresponding author at: Institute for Biomedical Engineering, University of Zurich and ETH Zurich, Wolfgang-Pauli-Strasse 27, 8093 Zurich, Switzerland.

E-mail address: klohs@biomed.ee.ethz.ch (J. Klohs).
} 
differences [15]. Thus, there is a general need for reference tissue values for interpreting QSM results.

Few studies have measured magnetic susceptibility of tissue itself $[16,17]$, but rather measurements of magnetic susceptibility have been made with purified biochemical materials that are believed to constitute the main determinants of susceptibility (e.g., iron, myelin, lipids, heme protein etc.) in the tissue [16,18-21]. In general, biological tissues are diamagnetic. For example, human tissue has a volume susceptibility between $-11.0 \times 10^{-6}$ to $-7.0 \times 10^{-6}$ [16]. This value is within $\pm 20 \%$ of the value for water, which has a mass susceptibility of water at $37{ }^{\circ} \mathrm{C}$ $(310 \mathrm{~K})$ of $-9.07 \times 10^{-9} \mathrm{~m}^{3} / \mathrm{kg}$ or a volume susceptibility of $-9.05 \times$ $10^{-6}[16-18]$ and which is the major component of tissue. Iron in its most common oxidation states $\left(\mathrm{Fe}^{2+}, \mathrm{Fe}^{3+}\right)$ and bound forms (e.g. heme proteins) is paramagnetic, which makes tissue containing iron less diamagnetic, whereas heavily myelinated tissue for example in the brain is more diamagnetic [16]. In addition, blood cells that perfuse tissue contribute to their susceptibility. The volume magnetic susceptibility of red blood cells depends on the oxidation state of iron in haemoglobin. Deoxygenated blood cells are more paramagnetic $\left(-6.52 \times 10^{-6}\right)$ when compared to oxygenated red blood cells $\left(-9.19 \times 10^{-6}\right)[16,22-24]$. Thus, a systematic determination of magnetic susceptibility of tissues and organs is required for interpreting QSM results.

Besides in vivo examinations, ex vivo MR susceptibility imaging of autopsy or biological samples is also often carried out [25-28]. Ex vivo studies allow for high spatial resolution MRI [29] and can guide pathological examinations, or can be combined with histopathology or other biochemical methods to identify underlying differences in tissues [25,30-32]. Tissues that undergo ex vivo MRI are usually subjected to chemical fixation, using (para-)formaldehyde (PFA) solution for tissue preservation $[33,34]$. The fixation procedure has many effects on the chemical composition and biophysical properties of tissue. A previous QSM study has shown no effects of chemical fixation on magnetic susceptibility of human brain tissue [28]. Thus, an independent validation of this observation and a more systemic investigation of the other tissue types has so far not been attempted, but remains important for comparing in vivo and ex vivo MR susceptibility imaging studies.

In the current study, we determined the mass susceptibility of fresh and fixed heart, brain liver and skeletal muscle tissue of mice, that are organs and tissue most often investigated with MR susceptibility imaging with a vibrating sample magnetometer (VSM). Measurements of water content of tissues was performed to establish influence on magnetic susceptibility. Susceptibly was obtained from measuring induced magnetization as a function of applied magnetic field. We have further observed the temperature dependence of susceptibility, which will indicate if the tissue is purely diamagnetic or if there is a contribution arising from a paramagnetic component.

\section{Methods}

\section{Animals and sample preparation}

All experiments were performed in accordance with the Swiss Federal Act on Animal Protection and approved by the Cantonal Veterinary Office Zurich (permit number: ZH044/19). Ten C57BI6/J mice (Janvier, France) were used that had a weight range from 20 to $25 \mathrm{~g}$ and an age range from 12 to 20 weeks. Animals were housed in individually ventilated cages inside a temperature-controlled room, under a 12-hour dark/light cycle. Pelleted food and water was provided ad-libitum.

Mice were deeply anesthetized by intraperitoneal injection of sodium pentobarbital (100 mg/kg body weight). To remove blood from circulation, mice were perfused intracardially with $10 \mathrm{ml}$ of $0.1 \mathrm{M}$ phosphate buffered saline (PBS, pH 7.4). The heart, liver, thigh muscles of both hind legs and the brain were subsequently removed from each animal. Tissues and organs were divided (except the heart for measurement of magnetic mass susceptibility, which would have rendered samples too small). One half was immersed in 4\% PFA in $0.1 \mathrm{M} \mathrm{PBS} \mathrm{(pH}$
7.4) for $24 \mathrm{~h}$. The other half was placed in a container, frozen on dry ice and kept frozen at $-20^{\circ} \mathrm{C}$ until further use.

\section{Magnetic mass susceptibility}

Volume magnetic susceptibility $(\chi)$ is a material constant that reflects the relationship to the induced magnetization $(M)$ that a material acquires in a weak applied field $(H)$ :

$M=\chi H$

Both $M$ and $H$ are measured in $\mathrm{A} / \mathrm{m}$ such that $\chi$ is without units. Magnetic mass susceptibility $\left(\chi_{\text {mass }}\right)$ was calculated by:

$\chi_{\text {mass }}=\chi / \rho$

where densities ( $\rho$ ) of the tissue were taken from [35]. The units for $\chi$ mass are in $\mathrm{m}^{3} / \mathrm{kg}$.

The magnetic susceptibility of the samples is determined from the slope of the induced $M$ of the sample in the applied DC field [36] when using a VSM. VSMs have been previously shown to yield accurate and reliable values of susceptibility of mineral inclusions, nanoparticles and biological tissue [37-39]. Measurements on tissue samples from 5 mice were made on a VSM (Model 3900, Princeton Measurements Corporation, $\mathrm{PCM})$ at room temperature $(295 \mathrm{~K})$ using a $250 \mathrm{~ms}$ averaging time and $2.55 \mathrm{kA} / \mathrm{m}$ sample interval between $\pm 637 \mathrm{kA} / \mathrm{m}$. A full magnetization curve was obtained within $500 \mathrm{~s}$. The instrument was calibrated with a Yt-Fe-garnet that was certified by the U.S. National Institute of Standards (NIST). For measurements, samples were inserted into a plastic straw (length $19.8 \mathrm{~cm}$, diameter $0.6 \mathrm{~cm}$ ) and gently pressed into the straw $(6 \mathrm{~mm})$ so that the height of sample was on the order of the width to preserve a dipole signal. The straw was sealed at the bottom end with hot glue. Supplementary Table 1 provides a list of tissue samples. Each holder was measured before the samples was inserted, so that its contribution to the total magnetization could be removed. The empty sample holder consisted of a plastic straw in which one end was sealed with glue. Frozen samples were allowed to thaw and reach room temperature for measurements. Note that the tissue samples were not measured in any particular order with respect to tissue type or whether the tissue was fresh or fixed tissue. A magnetization curve was also measured for three samples of double-distilled water and two samples of the PFA solution for comparison with the tissue samples. In this case, a glass capillary tubes with ca. $6 \mathrm{~mm}$ length and $5 \mathrm{~mm}$ diameter that were sealed on one end with epoxy was used for the liquid samples.

To assess the behavior of tissue susceptibilities, magnetization curves were measured on the VSM with a PCM cryostat at 50, 125, 200 and 295 $\mathrm{K}$. The same measurement parameters were used for the low temperature measurements. For low temperature, two samples of both fresh and fixed tissue from the brain, liver and muscle and one fresh and fixed heart samples were cooled to temperature until thermocouple showed that the region immediately below the sample had reached the set temperature. If there was any thermal drift, the magnetization was repeated. Note that the temperature within the cryostat varied by $\leq 1.0$ $\mathrm{K}$ during the measurement. Diamagnetic susceptibility is independent of temperature [40], but an empty holder was also measured to confirm that there was no change in susceptibility as a function of temperature.

\section{Measurement of water content}

We assessed fresh and fixed tissue samples from 5 mice. Samples were weighted (wet weight) after preparation and placed in an oven at $120^{\circ} \mathrm{C}$ for $50 \mathrm{~h}$. Samples for weighted again (dry weight). Water content (\%) was calculated as the percentage difference between wet and dry weights.

\section{Statistics}

Statistical analysis was performed using SigmaPlot Version 13 
(Systat Software Inc., CA, USA). A two-way ANOVA with Holm-Sidak's post-hoc analysis was used for comparison of magnetic susceptibilities of fresh and fixed tissues. The difference between groups was considered significant $(*)$ at $\mathrm{p}$ value $<0.05$.

\section{Results}

Biological tissue shows diamagnetic susceptibility

All holders showed a weak paramagnetic magnetization, where slight variations between holders were due to small differences in the length of the straws and the amount of glue. For this reason, each holder was measured before the samples was inserted, so that its contribution to the total magnetization could be removed. The frozen samples were allowed to thaw during sample preparation. Therefore, initial magnetization measurements were made at room temperature for both the fresh and fixed tissue. The magnetization for all fresh and fixed samples is weak, but shows a linear relation of their magnetization as a function of field (Fig. 1a). The magnetization curve of each sample was measured again after approximately $24 \mathrm{~h}$ to monitor for any change in susceptibility over time (data not shown). All measurements were reproducible which showed that the measurement of $\chi$ mass was repeatable. Based on the measurements the magnetic mass susceptibility for each sample was calculated (Supplementary Table 1) All samples show diamagnetic susceptibility between -0.068 and $-1.929 \times 10^{-8} \mathrm{~m}^{3} / \mathrm{kg}$. The sample of double distilled water has a diamagnetic susceptibility of $-9.338 \times 10^{-9}$ $\mathrm{m}^{3} / \mathrm{kg}$ (Table 1, Fig. 1b). Heart tissue has a higher susceptibility (i.e., more diamagnetic) than the other tissue samples.

\section{PFA fixation increases mass susceptibility}

We tested the effect of fixation on magnetic mass susceptibility (Table 1, Fig. 1b). Generally, less fixed than fresh tissue was available. PFA solution ( $4 \%$ in $0.1 \mathrm{M}$ PBS) has a susceptibility of $-4.966 \times 10^{-9}$ $\mathrm{m}^{3} / \mathrm{kg}$. Fixation made the sample less diamagnetic for the heart tissue. For all other organs there were not significant differences in magnetic mass susceptibility between fresh and fixed tissue. Notwithstanding, when we pooled values of organs we observed that the fixed tissue was less diamagnetic compared to fresh tissue.

Water content of fresh and fixed tissue

To assess the relation between water content and magnetic susceptibility of the tissue, we measured water content in fresh and fixed tissue samples (Table 2). For comparison we have also summarized water content for dog [41], rat [42,43], and human tissue [44-46]. Values for water content in mouse tissue was is in good agreement with literature values from the tissues of the other species. We also assessed the effect of chemical fixation on water content. Fixation changed water content by $-3.0,0.5,3.9$ and $3.3 \%$ in the heart, brain, liver and muscle respectively. We did not find statistically significant differences in the water content between fresh and fixed samples of all tissues.

\section{Paramagnetic contribution to magnetic mass susceptibility in fresh tissue}

Selected fresh and chemically fixed samples were measured at low temperature to establish if their susceptibility shows a temperature dependence. Note that diamagnetic susceptibility does not show a temperature dependence, whereas paramagnetic susceptibility, according to Curie's law, will show an inverse relationship on a first order [40]. Fresh samples of all tissue types show a small systematic change in susceptibility between $295 \mathrm{~K}$ and $125 \mathrm{~K}$ that makes it less diamagnetic, but a slight decrease at $50 \mathrm{~K}$ (Fig. 2). This behavior indicates a partial paramagnetic contribution to the mass susceptibility. Chemically fixed tissue does not show a systematic dependence of susceptibility with temperature.

\section{Discussion}

We determined the mass magnetic susceptibly of different mouse organs and skeletal muscle tissue with VSM. All measured tissue samples show diamagnetic susceptibility between -0.0675 and $-1.929 \times 10^{-8}$ $\mathrm{m}^{3} / \mathrm{kg}$ and points to the predominance of diamagnetic constituents in tissue. Heart tissue was found more diamagnetic to what has been reported in the literature, whereas for the other tissues values were slightly below what has been reported $[16,40]$. However, in one study [16] it was not clear how the reported values were measured. While water is diamagnetic and a major constituent of most biological tissues, the susceptibility of the tissue is not equal to the susceptibility of water $[16,18]$. We have not seen a relation between magnetic mass susceptibility and water content in fresh tissue. The heart tissue showed a more diamagnetic response compared to water and the other tissues showing a higher susceptibility compared to water, while also the brain has a similar water content than the heart. The more diamagnetic behavior of the heart tissue was noted in the unprocessed magnetic data before normalization by the sample mass, as seen from the steeper slope of the
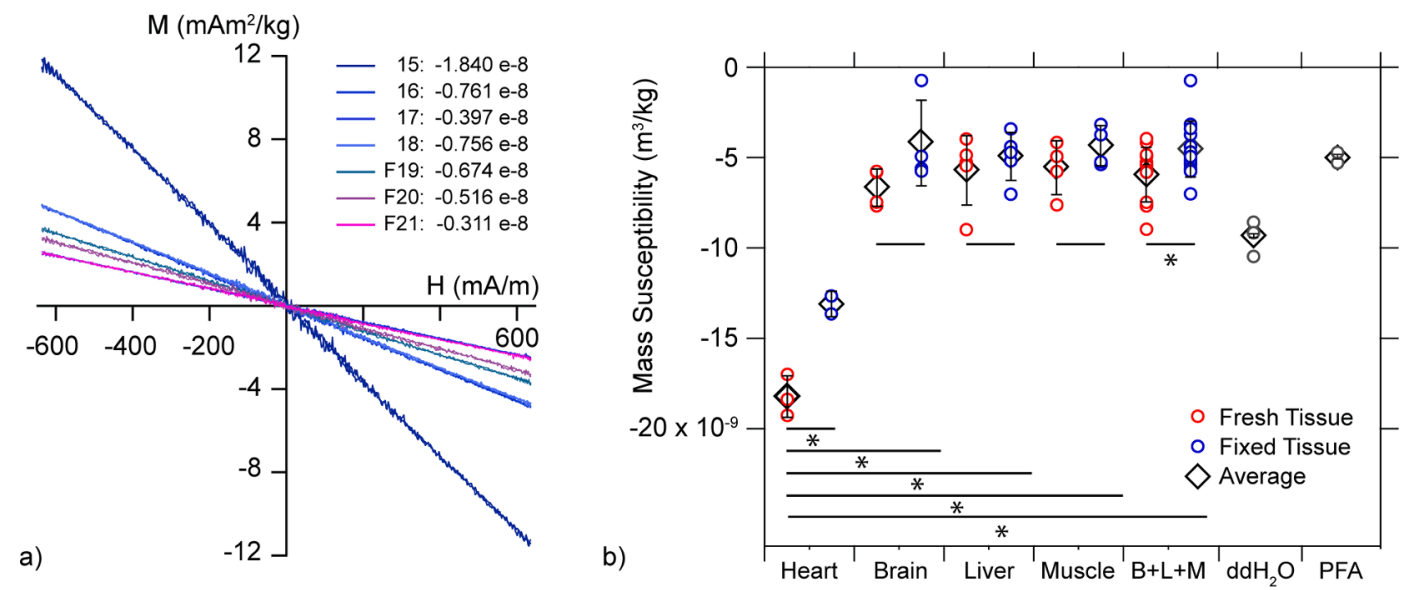

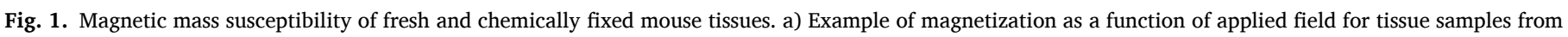

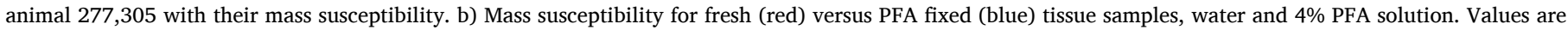

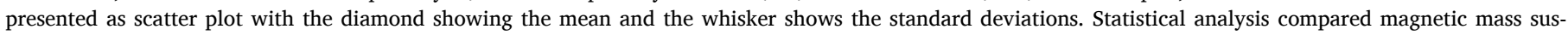

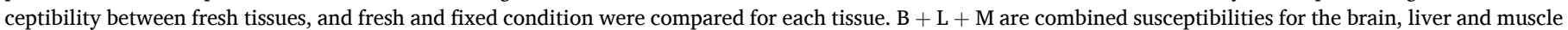
tissue together. (For interpretation of the references to colour in this figure legend, the reader is referred to the web version of this article.) 
Table 1

Magnetic mass susceptibility $\chi_{\text {mass }}$ of fresh and fixed tissues measured at $295.1 \pm 0.6 \mathrm{~K}\left(22.1{ }^{\circ} \mathrm{C}\right)$.

\begin{tabular}{|c|c|c|c|c|c|}
\hline Tissue & Condition & Density kg/m³ from [35] & $\begin{array}{l}\text { Mass magnetic susceptibility, } \chi_{\text {mass }}, \mathrm{m}^{3} / \mathrm{kg} \\
\text { (Volume magnetic susceptibility, } \chi \text { ) }\end{array}$ & $\%$ Change after fixation & Number of specimen \\
\hline \multirow[t]{2}{*}{ Heart } & Fresh & 1060 & $\begin{array}{l}-1.823 \pm 0.115 \times 10^{-8} \\
\left(-1.932 \times 10^{-5}\right)\end{array}$ & & 3 \\
\hline & Fixed & 1060 & $\begin{array}{l}-1.310 \pm 0.071 \times 10^{-8} \\
\left(-1.389 \times 10^{-5}\right)\end{array}$ & 28.1 & 2 \\
\hline \multirow[t]{2}{*}{ Brain } & Fresh & 1040 & $\begin{array}{l}-6.662 \pm 1.039 \times 10^{-9} \\
\left(-6.928 \times 10^{-6}\right)\end{array}$ & & 4 \\
\hline & Fixed & 1040 & $\begin{array}{l}-4.197 \pm 2.375 \times 10^{-9} \\
\left(-4.365 \times 10^{-6}\right)\end{array}$ & 37.0 & 4 \\
\hline \multirow[t]{2}{*}{ Liver } & Fresh & 1060 & $\begin{array}{l}-5.708 \pm 1.918 \times 10^{-9} \\
\left(-6.050 \times 10^{-6}\right)\end{array}$ & & 6 \\
\hline & Fixed & 1060 & $\begin{array}{l}-4.938 \pm 1.326 \times 10^{-9} \\
\left(-5.234 \times 10^{-6}\right)\end{array}$ & 13.5 & 4 \\
\hline \multirow[t]{2}{*}{ Muscle } & Fresh & 1040 & $\begin{array}{l}-5.559 \pm 1.488 \times 10^{-9} \\
\left(-5.781 \times 10^{-6}\right)\end{array}$ & & 4 \\
\hline & Fixed & 1040 & $\begin{array}{l}-4.334 \pm 1.107 \times 10^{-9} \\
\left(-4.507 \times 10^{-6}\right)\end{array}$ & 22.0 & 4 \\
\hline Double distilled water & & 1000 & $\begin{array}{l}-9.338 \pm 0.026 \times 10^{-9} \\
\left(-9.338 \times 10^{-6}\right)\end{array}$ & & 3 \\
\hline 4\% PFA solution & & 1000 & $\begin{array}{l}-4.966 \times 10^{-9} \\
\left(-4-966 \times 10^{-6}\right)\end{array}$ & & 2 \\
\hline
\end{tabular}

Table 2

Water content of fresh and fixed tissues (five specimens each condition) and comparison with the literature.

\begin{tabular}{lllll}
\hline Organ & Species & Condition & $\begin{array}{l}\text { Water content } \\
\%\end{array}$ & References \\
& Mouse & Fresh & $79.0 \pm 1.4$ & \\
\hline \multirow{2}{*}{ Heart } & Douse & Fixed & $76.6 \pm 3.5$ & \\
& Human & Fresh & $78.3 \pm 0.4$ & {$[41]$} \\
& Fresh & 80.0 & {$[44]$} \\
Brain (grey and white & Mouse & Fresh & $78.5 \pm 1.2$ & \\
matter) & & & & \\
& Mouse & Fixed & $78.9 \pm 0.4$ & \\
& Rat & Fresh & 75.8 & {$[42]$} \\
& Rat & Fresh & $77.2-78.6$ & {$[43]$} \\
Hiver & Human & Fresh & 77.5 & {$[44]$} \\
& Mouse & Fresh & $66.3 \pm 3.4$ & \\
& Mouse & Fixed & $68.9 \pm 1.5$ & \\
& Rat & Fresh & 77.3 & {$[42]$} \\
& Human & Fresh & 71.1 & {$[44]$} \\
& Mouse & Fresh & $72.0 \pm 0.5$ & \\
& Mouse & Fixed & $74.4 \pm 2.5$ & \\
& Human & Fresh & 79.2 & {$[44]$} \\
& Human & Fresh & $76.2 \pm 1.1$ & {$[45]$} \\
& Human & Fresh & $77 \pm 2$ & {$[46]$} \\
\hline
\end{tabular}

For measurements of water content, 5 specimens were investigated for each condition.

heart samples with respect to the sample mass, in comparison to other tissue samples (Supplementary Fig. 1). Because the heart samples were measured between measurements with other tissue samples, indicates that the difference is real and not an artefact of the experimental procedure.

In biological tissues, other biochemical constituents than water contribute to the net bulk susceptibility in a complex way. For example, it is known that lipids and structural proteins such as actin, collagen and myelin are diamagnetic $[16,18-21]$. But lipids are less diamagnetic than water and proteins [47]. They are highly abundant in some tissues e.g. $70 \%$ as myelin in the white matter of the brain [48], but less abundant in others, e.g. $12 \%$ in skeletal muscle [49]. However, as we have not investigated the determinants of magnetic susceptibility other than water for each tissue, we do not know which constituent contributes to the diamagnetic property of tissue. Moreover, anisotropy of magnetic susceptibility of proteins like collagen and myoglobin [50,51] would make VSM sensitive to the distribution of constituents in tissue of similar composition but different architecture [52]. However, these
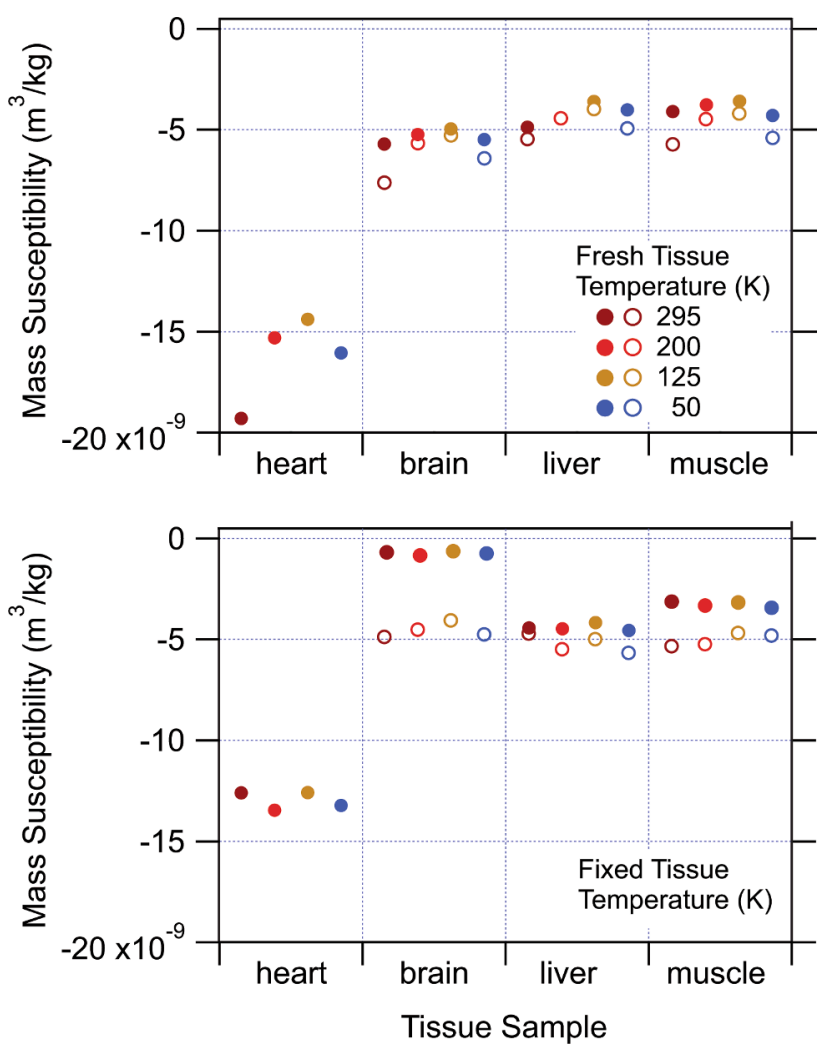

Fig. 2. Assessing temperature dependency of magnetic mass susceptibility. Magnetization curves were measured with a) fresh and b) chemically fixed tissue samples at 50, 125, 200 and $295 \mathrm{~K}$. Individual values are presented.

contributions are expected to be very small particularly because the tissue was not placed in the holders that would lead to a preferential orientation of proteins or myoglobin. More research would be needed with physically aligned elements, to understand the exact contribution of the microarchitecture to the tissue susceptibility.

The temperature dependence of susceptibility to at least $125 \mathrm{~K}$ in fresh tissue suggests that there is a paramagnetic contribution to the mass susceptibility. Ions, atoms, and molecules with unpaired spins provides a paramagnetic contribution to tissue susceptibility. The 
transition elements known to be present in biological tissue are iron, copper, manganese, nickel, chromium [20]. At physiological temperatures, paramagnetism is much more powerful than diamagnetism on an atom by atom basis [20]. A single paramagnetic molecule can cancel the diamagnetism of hundreds or thousands of water molecules [16]. We have removed blood, which would be mainly paramagnetic deoxygenated blood [16,22-24] in ex vivo tissue, by PBS perfusion prior dissection, thus have eliminated circulating sources of iron. However, iron is stored in tissue predominately in myoglobin and ferritin [53]. Myoglobin is largely found in skeletal and cardiac muscle [54], but under physiological conditions is has not be found in the brain [55]. Deoxymyoglobin is paramagnetic [50]. Thus, in the absence of hemoglobin, we think that we have paramagnetic contributions of these proteins to the magnetic susceptibility of the tissue. However, despite their strong effects paramagnetic elements have a comparably low concentration in tissue, thus rendering the tissue overall diamagnetic. This applies even to organ that are iron stores like the liver and spleen, where we found liver tissue to be diamagnetic. Even with excessive storage of iron in tissues that occurs in hereditary hemochromatosis the liver becomes only weakly paramagnetic [10]. Taken together, all tissues are diamagnetic, but are less diamagnetic than water due to the presence of other constituents with higher susceptibility, such as any paramagnetic constituent.

Tissue composition can vary considerably, depending on the species, organ and subregions, age, gender, genetics, and environmental factors (diets etc.) etc. We opted to use mouse tissue and not human autopsy tissue for the measurement where factors like age, gender, housing conditions as well as the timing and methods of tissue collection can be better controlled. It is widely assumed that biochemical and cellular composition in the mouse is highly conserved with humans, and thus the current findings might be extrapolated to human tissues [56]. It may be interesting to study effects of different factors such as age on susceptibilities of different tissues. For example, it is known that concentrations of paramagnetic iron in tissue can vary considerably with ageing in most tissues [57]. In the brain, iron concentration increases during early childhood and during ageing and are pathologically altered in neurodegenerative diseases $[58,59]$. Also the concentration of diamagnetic constituents such as myelin in the brain change during ageing and under pathological conditions [60]. To validate the diagnostic utility of use susceptibility-based MRI samples from pathological tissues should be investigated as a variety of diseases is associated with changes in tissue composition [10,58-60].

There are differences in volume magnetic susceptibility of mouse tissues measured ex vivo with VSM and values reported previously from QSM in vivo studies. For the brain volume magnetic susceptibilities between -0.003 to $0.2 \times 10^{-6}$, depending on the brain region, were reported $[6,27,61]$, which are lower than $-6.393 \times 10^{-6}$ obtained with VSM. A volume magnetic susceptibility $-9.11 \times 10^{-6}$ for the forearm muscle was reported, which is more diamagnetic than the value of $-5.781 \times 10^{-6}$ obtained here. Differences in values are likely, in addition to differences in samples (in vivo vs ex vivo, location, etc.) due to differences in measurements of susceptibility. With a VSM, the induced magnetization is measured that arises from the material that is being vibrated in an applied DC field. In QSM, bulk susceptibility is inferred from the phase evolution of the MRI signal [2-8]. While QSM is supposed to yield a physical tissue property, susceptibility maps calculated with different algorithm from the same dataset have shown substantial differences [15]. Moreover, while there is a good correlation between the observed tissue susceptibility on susceptibility maps with the concentrations of tissue constituents with strong magnetic moments such as iron $[27,28]$, the contribution of many other tissue constituents to the computed tissue susceptibility is largely not known and may not only rely on the electromagnetic properties of its components, but also their distribution in the tissue and the microarchitecture of the tissue $[47,62-65]$. Thus, there is a general need to better understand how susceptibility of tissue components give rise to variations in susceptibility maps in MRI.

Besides examinations in vivo, ex vivo MR susceptibility imaging of autopsy or biological samples is also often carried out [25-28]. Chemical fixation with PFA solution is ideally suited to prevent or arrest tissue autolysis, to maintain cellular and subcellular architecture of tissue and the local chemical composition, and to allow for long-term preservation of tissue [33]. In chemical fixation cross-links in and between proteins, in and between nucleic acids and between nucleic acids and proteins are formed. The process causes non-uniform shrinkage of tissue [66] and changes water diffusion properties [67-71]. It addition, chemical fixation shortens the $\mathrm{T}_{1}, \mathrm{~T}_{2}$ and $\mathrm{T}_{2} *$ relaxation times of tissues compared to their in vivo state [70-74]. A previous QSM study has observed no differences in the magnetic susceptibility of fresh and fixed human brain tissue [28], but validation of this finding and systematic measurements of the effect of fixation on magnetic susceptibility on other tissues are lacking.

We used immersion fixation of all tissues and found that the magnetic mass susceptibility of fixed tissue was less diamagnetic than the fresh tissue. This was more obvious for the heart and differences were smaller for the other tissues, and were only significantly different upon pooling of values. To test if these changes are due to effects of changes in water content, we measured water content in fresh and fixed tissue samples. Interestingly, we did not find significant changes in water content between fresh and fixed tissue. Similar to the fresh tissue, magnetic susceptibility was not mirrored by the water content of the fixed tissue. Paraformaldehyde has both a paramagnetic and diamagnetic contribution $[74,75]$. A paramagnetic effect of formalin was reported previously reported [74]. Here, we found that 4\% PFA solutions is less diamagnetic than water. Thus, it is likely that the presence of paraformaldehyde has led to the observed small shift in the susceptibility of tissue upon fixation. Moreover, tissue fixation is accompanied by changes such as shrinkage, swelling and hardening of tissue components, and thus affecting the magnetic microarchitecture. Effect of fixation manifests differently in different tissues. Pathological studies and studies using diffusion tensor imaging have shown profound changes in morphology and microstructure (e.g. shifts in fiber orientation) in the heart upon chemical fixation with PFA solution [67-69]. For the brain mixed results on the effect of fixation on microstructural changes have been reported $[70,71,76-78]$. Thus, cross-linking of proteins and changes in microstructure might have additional effects on tissue susceptibility. We have not tested for fixation intervals longer than $24 \mathrm{~h}$. Given the speed of fixation of $1 \mathrm{~mm}$ tissue in one hour [33] and the tissue thickness of approximately $3-5 \mathrm{~mm}$, all tissue was expected to be fixed completely in the chosen interval. However, long-term storage in the fixative can lead to dissolution and/or leaching of transition elements [79,80], which may significantly alter tissue susceptibility.

In the current study, we used VSM to determine magnetic mass susceptibility in biological tissue samples from the mouse. One advantage to measurements on the PCM VSM is the rapidity of measurement, compared to other systems that measure induce magnetization in an applied magnetic field. This has the advantage that less temperature variation and chemical alteration can occur within the (fresh) tissue during the measurement. Moreover, VSM has a high sensitivity and can be used to determine weak susceptibilities of tissues and solution with a high reproducibility. The instrument accuracy was verified from the determination of $\chi$ mass of distilled water, which is $<3 \%$ difference with the theoretical value of pure water of $-9.07 \times 10^{-9} \mathrm{~m}^{3} / \mathrm{kg}[16,18]$. Note that this difference is on the order of the sensitivity range of the instrument, therefore any slight variation in the susceptibility of the sample holder between measuring the empty state and filling, or a slight difference in positioning could account for the small variation.

The current study has a few limitations. 1) We measured the mass magnetic susceptibility of fresh tissue ex vivo, which may differ from the susceptibility of tissue in vivo. Susceptibilities of heme proteins in tissue depend on oxygen saturation $[16,22-24,50]$ and might thus differ 
between in vivo from ex vivo conditions, where proteins are deoxygenated. In vivo VSM measurements are not feasible and notwithstanding these limitations, ex vivo VSM investigations have provided reference values for magnetic susceptibilities of different tissues. 2) We have removed blood prior dissection of the tissue by extensive PBS perfusion. We reasoned that the fixation procedure might remove blood from the tissue in an inhomogeneous way, introducing variability. Furthermore, we would not have the same amount of hematocrit in fresh and fixed samples. However, the removal of blood affects vascular organs with a high blood volume like the liver or heart more strongly than organs that have a lower blood volume such as the brain. Thus, we do not know the contribution of blood to the tissue susceptibility under in vivo conditions. 3) The time lapse between animal sacrifice and tissue immersion in PFA solution was insignificant, and tissue autolysis was prevented. For the fresh tissue some tissue degradation may have started with thawing of the sample and the effects on the measurements are not known. 4) We measured mass magnetic susceptibilities of organs and tissues at room temperature as it was not possible to heat the sample to body temperature i.e. $36.5 \pm 0.5{ }^{\circ} \mathrm{C}$. As paramagnetic susceptibility is dependent on temperature there might be differences between susceptibility of fresh tissue and tissue in vivo, but due to the small difference in temperature between the two conditions and the low concentration of paramagnetic elements in tissue, this difference is expected to be small. 5) We used densities for fresh tissue for the calculation of volume magnetic susceptibilities, though fixation might alter tissue densities. 6) The low sample sizes for same tissues like the heart, and the measurement of the temperature dependency of susceptibility. 7) Lastly, we only measured bulk magnetic mass susceptibility of organs and tissue. Brain tissue but also skeletal muscle are known to have susceptibility variations across the tissue. However, the measured sample volumes were on the limit in terms of signal strength, i.e., trying to isolate individual parts of organs e.g. specific brain regions or certain muscle fibers would leave too little material for reliable measurement of susceptibility values. Future studies could investigate regional variations in tissues e.g. the brain, determine biochemical underpinnings, and study oriental effects of tissue magnetic susceptibility.

\section{Conclusions}

We showed with VSM data that biological tissues are diamagnetic in comparison to water with paramagnetic contribution, where the heart is more diamagnetic than the other tissues. Fixation rendered tissue less diamagnetic compared to fresh tissue. The magnetic susceptibility of fresh and fixed tissues is not mirrored by its water content. VSM measurements revealed differences in tissue magnetic susceptibility of biological tissue obtained with QSM in vivo, inviting more research to compare susceptibility-based MRI methods with physical measurements of tissue susceptibility.

\section{Declaration of Competing Interest}

The authors declare that they have no known competing financial interests or personal relationships that could have appeared to influence the work reported in this paper.

\section{Acknowledgements}

JK received funding from the Swiss National Science Foundation (320030_179277), ERA-NET NEURON (32NE30_173678/1), the Olga Mayenfisch Stiftung, the Synapsis foundation and the Vontobel foundation. We acknowledge the help of Dr. Evi and Prof. Katrien de Bock, Laboratory of Exercise and Health, Department of Health Sciences and Technology, ETH Zurich, Zurich, Switzerland, for drying of the tissue samples. In addition, we thank the reviewers of the manuscript for their thorough evaluation and very valuable suggestions.

\section{Appendix A. Supplementary data}

Supplementary data to this article can be found online at https://doi. org/10.1016/j.ejmp.2021.06.014.

\section{References}

[1] Reichenbach JR, Venkatesan R, Schillinger DJ, Kido DK, Haacke EM. Small vessels in the human brain: MR venography with deoxyhemoglobin as an intrinsic contrast agent. Radiology 1997;204:272-7. https://doi.org/10.1148/ radiology.204.1.9205259.

[2] Li L, Leigh JS. Quantifying Arbitrary Magnetic Susceptibility Distributions with MR. Magn Reson Med 2004;51:1077-82. https://doi.org/10.1002/mrm.20054.

[3] Duyn JH, Schenck J. Contributions to magnetic susceptibility of brain tissue. NMR Biomed 2017;30. https://doi.org/10.1002/nbm.3546.

[4] Liu T, Spincemaille P, De Rochefort L, Kressler B, Wang Y. Calculation of susceptibility through multiple orientation sampling (COSMOS): A method for conditioning the inverse problem from measured magnetic field map to susceptibility source image in MRI. Magn Reson Med 2009. https://doi.org/ $10.1002 / \mathrm{mrm} .21828$.

[5] Haacke EM, Tang J, Neelavalli J, Cheng YCN. Susceptibility mapping as a means to visualize veins and quantify oxygen saturation. J Magn Reson Imaging 2010. https://doi.org/10.1002/jmri.22276.

[6] Wharton S, Bowtell R. Whole-brain susceptibility mapping at high field: A comparison of multiple- and single-orientation methods. Neuroimage 2010. https://doi.org/10.1016/j.neuroimage.2010.06.070.

[7] Li W, Wu B, Liu C. Quantitative susceptibility mapping of human brain reflects spatial variation in tissue composition. Neuroimage 2011. https://doi.org/ 10.1016/j.neuroimage.2010.11.088.

[8] Schweser F, Deistung A, Lehr BW, Reichenbach JR. Quantitative imaging of intrinsic magnetic tissue properties using MRI signal phase: An approach to in vivo brain iron metabolism? Neuroimage 2011. https://doi.org/10.1016/j. neuroimage.2010.10.070.

[9] Klohs J, Deistung A, Schweser F, Grandjean J, Dominietto M, Waschkies C, et al. Detection of Cerebral Microbleeds with Quantitative Susceptibility Mapping in the Arcabeta Mouse Model of Cerebral Amyloidosis. J Cereb Blood Flow Metab 2011; 31:2282-92. https://doi.org/10.1038/jcbfm.2011.118.

[10] Sharma SD, Fischer R, Schoennagel BP, Nielsen P, Kooijman H, Yamamura J, et al. MRI-based quantitative susceptibility mapping (QSM) and R2* mapping of liver iron overload: Comparison with SQUID-based biomagnetic liver susceptometry. Magn Reson Med 2017;78:264-70. https://doi.org/10.1002/mrm.26358.

[11] Straub S, Schneider TM, Emmerich J, Freitag MT, Ziener CH, Schlemmer HP, et al. Suitable reference tissues for quantitative susceptibility mapping of the brain. Magn Reson Med 2017;78:204-14. https://doi.org/10.1002/mrm.26369.

[12] Vaas M, Deistung A, Reichenbach JR, Keller A, Kipar A, Klohs J. Vascular and Tissue Changes of Magnetic Susceptibility in the Mouse Brain After Transient Cerebral Ischemia. Transl Stroke Res 2018;9:426-35. https://doi.org/10.1007/ s12975-017-0591-X.

[13] Wen Y, Weinsaft JW, Nguyen TD, Liu Z, Horn EM, Singh H, et al. Free breathing three-dimensional cardiac quantitative susceptibility mapping for differential cardiac chamber blood oxygenation - Initial validation in patients with cardiovascular disease inclusive of direct comparison to invasive catheterization. J Cardiovasc Magn Reson 2019;21:1-13. https://doi.org/10.1186/s12968-0190579-7.

[14] Vinayagamani S, Sheelakumari R, Sabarish S, Senthilvelan S, Ros R, Thomas B, et al. Quantitative Susceptibility Mapping: Technical Considerations and Clinical Applications in Neuroimaging. J Magn Reson Imaging 2020. https://doi.org/ 10.1002/jmri.27058.

[15] Wang Y, Liu T. Quantitative susceptibility mapping (QSM): Decoding MRI data for a tissue magnetic biomarker. Magn Reson Med 2015. https://doi.org/10.1002/ mrm. 25358.

[16] Schenck JF. The role of magnetic susceptibility in magnetic resonance imaging: MRI magnetic compatibility of the first and second kinds. Med Phys 1996;23: 815-50. https://doi.org/10.1118/1.597854.

[17] Senftle BFE, States U, Survey G. April 29, 1961 1961;190:410-3.

[18] Arrighini GP, Maestro M, Moccia R. Magnetic properties of polyatomic molecules. I. Magnetic susceptibility of H2O, NH3, CH4, H2O2. J Chem Phys 1968;49:882-9. https://doi.org/10.1063/1.1670155.

[19] Savicki JP, Lang G, Ikeda-Saito M. Magnetic susceptibility of oxy- and carbonmonoxyhemoglobins. Proc Natl Acad Sci U S A 1984;81:5417-9. https:// doi.org/10.1073/pnas.81.17.5417.

[20] Schenck JF. Health and Physiological Effects of Human Exposure to Whole-Body Four-Tesla Magnetic Fields during MRI. Ann N Y Acad Sci 1992;649:285-301. https://doi.org/10.1111/j.1749-6632.1992.tb49617.x.

[21] Yablonskiy DA, Sukstanskii AL. Effects of biological tissue structural anisotropy and anisotropy of magnetic susceptibility on the gradient echo MRI signal phase: theoretical background. NMR Biomed 2017;30. https://doi.org/10.1002/ nbm.3655.

[22] Spees WMW, Da Yablonskiy DA, Oswood MC, Ackerman JJH. Water proton MR properties of human blood at 1.5 Tesla: Magnetic susceptibility, T1, T2, T2*, and non Lorentzian signal behavior. Magn Reson 2001;45:533-42. https://doi.org/ 10.1002/mrm.1072.

[23] Jain V, Abdulmalik O, Propert KJ, Wehrli FW. Investigating the magnetic susceptibility properties of fresh human blood for noninvasive oxygen saturation 
quantification. Magn Reson Med 2012;68:863-7. https://doi.org/10.1002/ mrm.23282.

[24] Plyavin' YA, Blum EY. Magnetic parameters of blood cells and high-gradient paramagnetic and diamagnetic phoresis. Magnetohydrodynamics 1983;19:349-59.

25] Wisnieff C, Ramanan S, Olesik J, Gauthier S, Wang Y, Pitt D. Quantitative susceptibility mapping (QSM) of white matter multiple sclerosis lesions: Interpreting positive susceptibility and the presence of iron. Magn Reson Med 2015;74:564-70. https://doi.org/10.1002/mrm.25420.

[26] Evia AM, Kotrotsou A, Tamhane AA, Dawe RJ, Kapasi A, Leurgans SE, et al. Ex-vivo quantitative susceptibility mapping of human brain hemispheres. PLoS ONE 2017; 12:1-16. https://doi.org/10.1371/journal.pone.0188395.

[27] Langkammer C, Schweser F, Krebs N, Deistung A, Goessler W, Scheurer E, et al. Quantitative susceptibility mapping (QSM) as a means to measure brain iron? A post mortem validation study. Neuroimage 2012;62:1593-9. https://doi.org/ 10.1016/j.neuroimage.2012.05.049.

[28] Deistung A, Endmayr V, Hametner S, Prihoda M, Feng X, Lassmann H, et al. Investigation of Brain Tissue Fixation on Iron Concentration, Magnetic Susceptibility and E ective Transverse Relaxation Rate. Proc. Intl. Soc. Mag. Reson. Med. 2017;25:3686.

[29] Wei H, Xie L, Dibb R, Li W, Decker K, Zhang Y, et al. Imaging whole-brain cytoarchitecture of mouse with MRI-based quantitative susceptibility mapping. Neuroimage 2016;137:107-15. https://doi.org/10.1016/j neuroimage.2016.05.033.

[30] O'Callaghan J, Holmes H, Powell N, Wells JA, Ismail O, Harrison IF, et al. Tissue magnetic susceptibility mapping as a marker of tau pathology in Alzheimer's disease. Neuroimage 2017;159:334-45. https://doi.org/10.1016/j. neuroimage.2017.08.003.

[31] Argyridis I, Li W, Johnson GA, Liu C. Quantitative magnetic susceptibility of the developing mouse brain reveals microstructural changes in the white matter. Neuroimage 2014;88:134-42. https://doi.org/10.1016/j. neuroimage.2013.11.026.

[32] Yu L, Dawe RJ, Boyle PA, Gaiteri C, Yang J, Buchman AS, et al. Association between brain gene expression, DNA methylation, and alteration of ex vivo magnetic resonance imaging transverse relaxation in late-life cognitive decline. JAMA Neurol 2017;74:1473-80. https://doi.org/10.1001/jamaneurol.2017.2807.

[33] Eltoum I, Fredenburgh J, Myers RB, Grizzle WE. Introduction to the theory and practice of fixation of tissues. J Histotechnol 2001;24:173-90. https://doi.org/ 10.1179/his.2001.24.3.173.

[34] Massalimova A, Ni R, Nitsch RM, Reisert M, von Elverfeldt D, Klohs J. Diffusion Tensor Imaging Reveals Whole-Brain Microstructural Changes in the P301L Mouse Model of Tauopathy. Neurodegener Dis 2021:1-12. https://doi.org/10.1159/ 000515754.

[35] Boutaleb S, Pouget JP, Hindorf C, Pèlegrin A, Barbet J, Kotzki PO, et al. Impact of mouse model on preclinical dosimetry in targeted radionuclide therapy. Proc IEEE 2009. https://doi.org/10.1109/JPROC.2009.2026921.

[36] Foner S. The vibrating sample magnetometer: Experiences of a volunteer (invited) J Appl Phys 1996. https://doi.org/10.1063/1.361657.

[37] Brem F, Hirt AM, Winklhofer M, Frei K, Yonekawa Y, Wieser HG, et al. Magnetic iron compounds in the human brain. J R Soc Interface 2006.

[38] Lemal P, Balog S, Ackermann-Hirschi L, Taladriz-Blanco P, Hirt AM, RothenRutishauser B, et al. Simple and fast evaluation of relaxation parameters of magnetic nanoparticles. J Magn Magn Mater 2020;499:166176. https://doi.org/ 10.1016/j.jmmm.2019.166176.

[39] Biedermann AR, Pettke T, Reusser E, Hirt AM. Anisotropy of magnetic susceptibility in natural olivine single crystals. Geochemistry, Geophys Geosystems 2014. https://doi.org/10.1002/2014GC005386.

[40] Senftle FE, Hambright WP. Magnetic Susceptibility of Biological Materials. Biol Eff Magn Fields 1969:261-306. https://doi.org/10.1007/978-1-4684-8352-9 19.

[41] Salisbury PF, Cross CE, Rieben PA. Distensibility and Water Content of Hear Muscle Before and After Injury. Circ Res 1960;8:788-93. https://doi.org/10.1161/ 01.RES. 8.4.788.

[42] Lee K, Jeoung K, Kim SH, Ji Y, Son H, Choi Y, et al. Measuring water contents in animal organ tissues using terahertz spectroscopic imaging. Biomed Opt Express 2018. https://doi.org/10.1364/boe.9.001582.

[43] Keep RF, Hua Y, Xi G. Brain Water Content: A Misunderstood Measurement? Trans Stroke Res 2012. https://doi.org/10.1007/s12975-012-0152-2.

[44] Mansfield P. Imaging by nuclear magnetic resonance. J Phys E Sci Instruments $1988 ; 21: 18-30$

[45] Hargens AR, Tipton CM, Gollnick PD, Mubarak SJ, Tucker BJ, Akeson WH. Fluid shifts and muscle function in humans during acute simulated weightlessness. J Appl Physiol Respir Environ Exerc Physiol 1983;54:1003-9. https://doi.org/ 10.1152/jappl.1983.54.4.1003.

[46] Ward SR, Lieber RL. Density and hydration of fresh and fixed human skeletal muscle. J Biomech 2005;38:2317-20. https://doi.org/10.1016/j biomech.2004.10.001.

[47] He X, Yablonskiy D, a.. Biophysical mechanisms of phase contrast in gradient echo MRI. Proc Natl Acad Sci U S A 2009;106:13558-63. https://doi.org/10.1073/ pnas.0904899106.

[48] Baumann N, Pham-Dinh D. Biology of oligodendrocyte and myelin in the mammalian central nervous system. Physiol Rev 2001;81:871-927. https://doi org/10.1152/physrev.2001.81.2.871.

[49] Masoro EJ, Rowell LB, McDonald RM, Steiert B. Skeletal muscle lipids. II. Nonutilization of intracellular lipid esters as an energy source for contractile activity. J Biol Chem 1966.
[50] Bertini I, Luchinat C, Turano P, Battaini G, Casella L. The magnetic properties of myoglobin as studied by NMR spectroscopy. Chem - A Eur J 2003;9:2316-22. https://doi.org/10.1002/chem.200204562.

[51] Worcester DL. Structural origins of diamagnetic anisotropy in proteins. Proc Natl Acad Sci U S A 1978;75:5475-7. https://doi.org/10.1073/pnas.75.11.5475.

[52] Arnold W, Steele R, Mueller H. On the magnetic asymmetry of muscle fibers. Proc Natl Acad Sci 1958;44:1-4.

[53] Finazzi D, Arosio P. Biology of ferritin in mammals: an update on iron storage, oxidative damage and neurodegeneration. Arch Toxicol 2014;88:1787-802. https://doi.org/10.1007/s00204-014-1329-0.

[54] Möller P, Sylvén C. Myoglobin in human skeletal muscle. Scand J Clin Lab Invest 1981;41:479-82. https://doi.org/10.3109/00365518109090486.

[55] Xie LK, Yang SH. Brain globins in physiology and pathology. Med Gas Res 2016;6: 154-63. https://doi.org/10.4103/2045-9912.191361.

[56] Treuting PM, Dintzis SM. Comparative Anatomy and Histology: A Mouse and Human Atlas. Atlas: Comp. Anat. Histol. A Mouse Hum; 2012.

[57] Xu J, Jia Z, Knutson MD, Leeuwenburgh C. Impaired iron status in aging research. Int J Mol Sci 2012. https://doi.org/10.3390/ijms13022368.

[58] Hallgren B, Sourander P. The effect of age on the non-haemin iron in the huma brain. J Neurochem 1958;3:41-51. https://doi.org/10.1111/j.1471-4159.1958. tb12607.x.

[59] Rouault TA. Iron metabolism in the CNS: Implications for neurodegenerative diseases. Nat Rev Neurosci 2013;14:551-64. https://doi.org/10.1038/nrn3453.

[60] Peters A. The effects of normal aging on myelin and nerve fibers: A review. J Neurocytol 2002. https://doi.org/10.1023/A:1025731309829.

[61] Bilgic B, Pfefferbaum A, Rohlfing T, Sullivan EV, Adalsteinsson E. MRI estimates of brain iron concentration in normal aging using quantitative susceptibility mapping. Neuroimage 2012;59:2625-35. https://doi.org/10.1016/. neuroimage.2011.08.077.

[62] Dibb R, Qi Y, Liu C. Magnetic susceptibility anisotropy of myocardium imaged by cardiovascular magnetic resonance reflects the anisotropy of myocardial filament $\alpha$ -helix polypeptide bonds. J Cardiovasc Magn Reson 2015;17:1-14. https://doi org /10.1186/s12968-015-0159-4.

[63] Li W, Wu B, Avram AV, Liu C. Magnetic susceptibility anisotropy of human brain in vivo and its molecular underpinnings. Neuroimage 2012;59:2088-97. https://doi. org/10.1016/j.neuroimage.2011.10.038.

[64] Kaden E, Gyori NG, Rudrapatna SU, Barskaya IY, Dragonu I, Does MD, et al. Microscopic susceptibility anisotropy imaging. Magn Reson Med 2020;84: 2739-53. https://doi.org/10.1002/mrm.28303.

[65] Luo J, He X, Yablonskiy DA. Magnetic susceptibility induced white matter MR signal frequency shifts - Experimental comparison between Lorentzian sphere and generalized Lorentzian approaches. Magn Reson Med 2014;71:1251-63. https:// doi.org/10.1002/mrm.24762.

[66] Schulz G, Crooijmans HJA, Germann M, Scheffler K, Müller-Gerbl M, Müller B. Three-dimensional strain fields in human brain resulting from formalin fixation. J Neurosci Methods 2011;202:17-27. https://doi.org/10.1016/j. jeumeth.2011.08.031.

[67] Hales PW, Burton RAB, Bollensdorff C, Mason F, Bishop M, Gavaghan D, et al. Progressive changes in T1, T2and left-ventricular histo-architecture in the fixed and embedded rat heart. NMR Biomed 2011;24:836-43. https://doi.org/10.1002/ nbm.1629.

[68] Giannakidis A, Gullberg GT, Pennell DJ, Firmin DN. Value of Formalin Fixation for the Prolonged Preservation of Rodent Myocardial Microanatomical Organization: Evidence by MR Diffusion Tensor Imaging. Anat Rec 2016;299:878-87. https:// doi.org/10.1002/ar.23359.

[69] Hołda MK, Klimek-Piotrowska W, Koziej M, Piątek K, Hołda J. Influence of different fixation protocols on the preservation and dimensions of cardiac tissue. $\mathrm{J}$ Anat 2016;229:334-40. https://doi.org/10.1111/joa.12469.

[70] Shepherd TM, Flint JJ, Thelwall PE, Stanisz GJ, Mareci TH, Yachnis AT, et al. NeuroImage Postmortem interval alters the water relaxation and diffusion properties of rat nervous tissue - Implications for MRI studies of human autopsy samples. Neuroimage 2009;44:820-6. https://doi.org/10.1016/j. neuroimage.2008.09.054.

[71] Shepherd TM, Thelwall PE, Stanisz GJ, Blackband SJ. Aldehyde Fixative Solutions Alter the Water Relaxation and Diffusion Properties of Nervous Tissue. Magn Reson Med 2009;34:26-34. https://doi.org/10.1002/mrm.21977.

[72] Kamman R, Go K, Stomp G, Hulstaert C, Berendsen H. Changes of relaxation times T1 and T2 in rat tissues after biopsy and fixation. Magn Reson Imaging 1985;3: 245-50. https://doi.org/10.1016/0730-725X(85)90353-4.

[73] Birkl C, Langkammer C, Golob-Schwarzl N, Leoni M, Haybaeck J, Goessler W, et al. Effects of formalin fixation and temperature on MR relaxation times in the human brain. NMR Biomed 2016;29:458-65. https://doi.org/10.1002/nbm.3477.

[74] Birkl C, Soellradl M, Toeglhofer AM, Krassnig S, Leoni M, Pirpamer L, et al. Effects of concentration and vendor specific composition of formalin on postmortem MRI of the human brain. Magn Reson Med 2018;79:1111-5. https://doi.org/10.1002/ mrm.26699.

[75] Flygare WH. Molecular Magnetic Moments and Susceptibility in Formaldehyde. J Chem Phys 1965;42:1563-8. https://doi.org/10.1063/1.1696162.

[76] Dyrby TB, Baaré WFC, Alexander DC, Jelsing J, Garde E, Søgaard LV. An ex vivo imaging pipeline for producing high-quality and high-resolution diffusionweighted imaging datasets. Hum Brain Mapp 2011;32:544-63. https://doi.org/ 10.1002/hbm.21043.

[77] Shatil AS, Uddin MN, Matsuda KM, Figley CR. Quantitative Ex Vivo MRI Changes due to Progressive Formalin Fixation in Whole Human Brain Specimens: Longitudinal Characterization of Diffusion, Relaxometry, and Myelin Water 
Fraction Measurements at 3T. Front Med 2018;5:1-15. https://doi.org/10.3389/ fmed.2018.00031.

[78] Yong-hing CJ, Obenaus A, Stryker R, Tong K, Sarty GE. Magnetic Resonance Imaging and Mathematical Modeling of Progressive Formalin Fixation of the Human Brain. Magn Reson Med 2005;332:324-32. https://doi.org/10.1002/ mrm. 20578.
[79] Gellein K, Flaten TP, Erikson KM, Aschner M, Syversen T. Leaching of trace elements from biological tissue by formalin fixation. Biol Trace Elem Res 2008;121: 221-5. https://doi.org/10.1007/s12011-007-8051-1.

[80] Dobson J, Grassi P. Magnetic properties of human hippocampal tissue - Evaluation of artefact and contamination sources. Brain Res Bull 1996;39:255-9. https://doi. org/10.1016/0361-9230(95)02132-9. 